\title{
Non-linear projection of the retinal image in a wide-angle schematic eye
}

\author{
N. DRASDO AND C. W. FOWLER \\ From the Department of Ophthalmic Optics, University of Aston in Birmingham
}

The non-linear projection of the retinal image has significant implications in both clinical ophthalmology and physiological optics, and was therefore considered by several early investigators, whose work culminated in that of Stine (1934), who obtained quantitative results by calculation. In an unpublished study on the Hering Hillebrand Horopter Deviation, it became necessary to re-evaluate this property of the retinal image in the light of contemporary knowledge of ocular dimensions, and some information of a wider relevance has emerged.

Many schematic eyes have been proposed as a model of the eye's optical function. These vary in complexity from the simple reduced eye of Listing (1853) to the recent example proposed by Pomerantzeff, Govignon, and Schepens (I97I), which has a lens cortex with 98 layers. It seemed possible, however, that such complexity was unnecessary for the purpose of the present study and that a simpler yet adequate model might be devised.

\section{Method}

Assembled data from review articles (Duke-Elder and Wybar, I96 I ; Duke-Elder and Abrams, I970) and individual reports (Prechtel and Wesley, 1970; Mandell and St Helen, 197I) were considered in an attempt to formulate an adequate specification for a simplified schematic eye, and this was found for the most part to approximate to the earlier paraxial data of Cowan (1928) which was utilized in a wide-angle model by Stine (1934). It appeared necessary, however, to make some fundamental modifications. Accumulating data on corneal topography has supported the suggestion of Helmholtz ( 1866 ) that the shape of the cornea is closely simulated by an ellipsoid. Such a surface can be specified in terms of an apical radius and an ' $e$ ' value, or eccentricity. The data of Prechtel and Wesley (1970), based on 150 eyes, show a clear unimodal tendency of this parameter which is further supported by the more detailed findings of Mandell and St Helen (1971) on eight human eyes. The peak value from these papers was taken as $e=0.5$, while retaining the apical radius of $7.8 \mathrm{~mm}$. which results in the ellipsoid defined by the simple equation:

$$
x=\sqrt{243 \cdot 3^{6-2 y^{2}}}
$$

It was also considered necessary to modify the data on the margin of the retina. This was located in accordance with the anatomical values quoted by Duke-Elder and Wybar (196I) on limbal distance of the ora serrata and the junction of the pars caeca with the functional retina. The resultant vertical and horizontal asymmetry of the retinal margin is shown in the Table, and is apparent also in the diagram of the optical geometry shown in Fig. I. The slope of the ellipse at any one point could be 
Table Data on simplified anatomical schematic eye Optical constants and dimensions

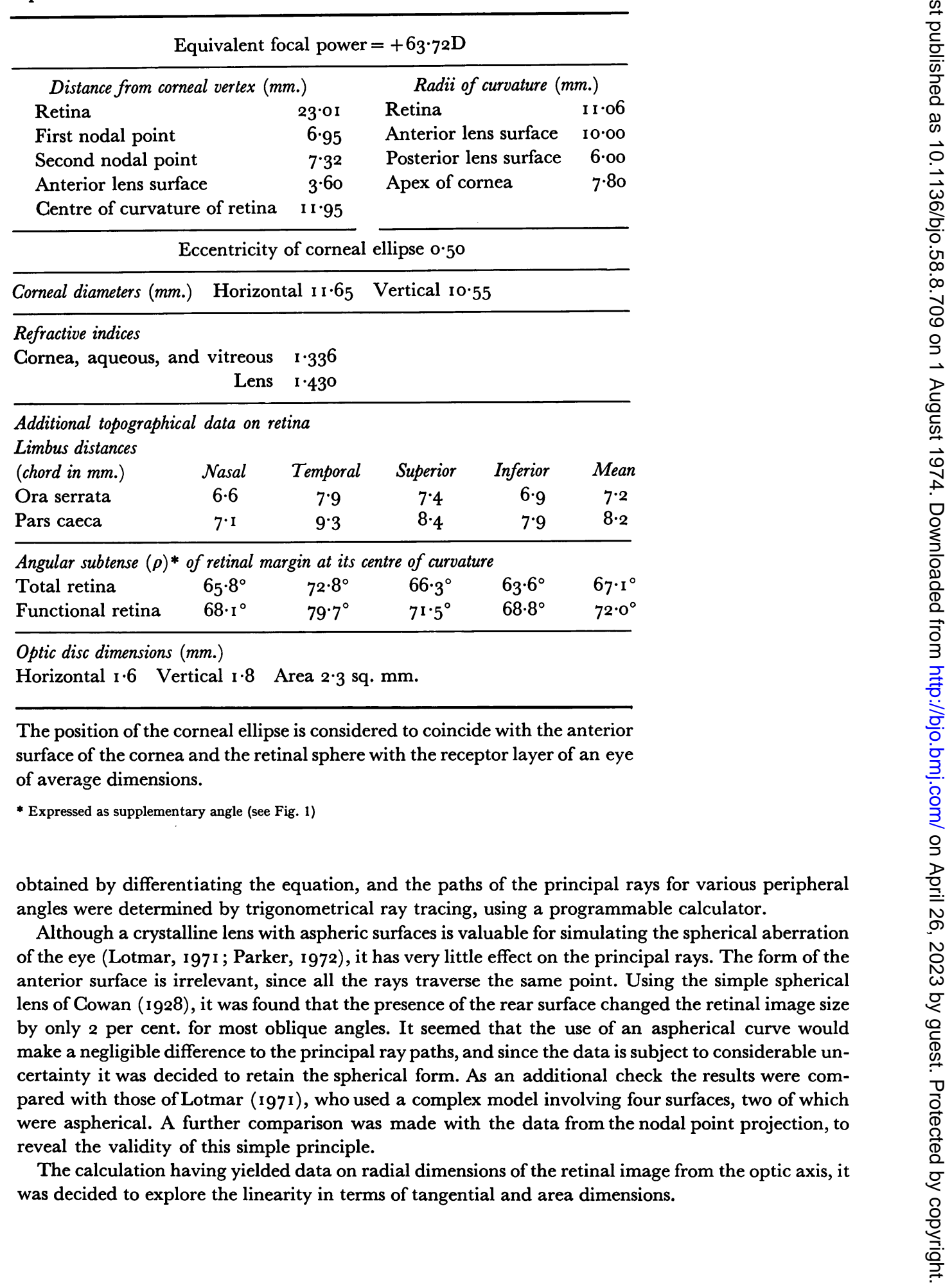




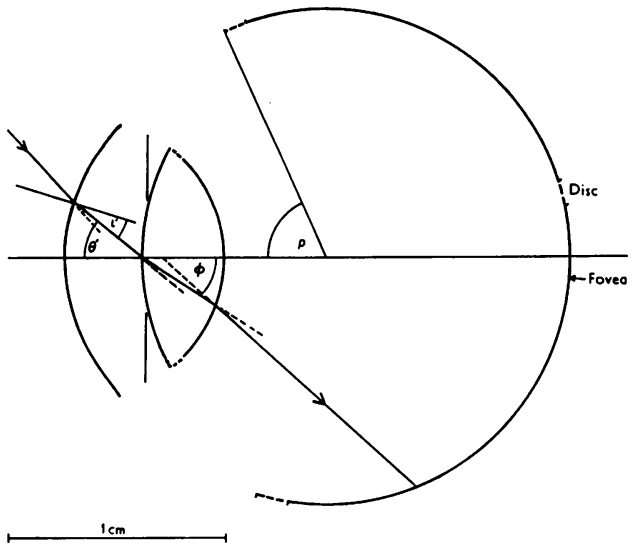

$$
\begin{aligned}
& \begin{array}{l}
\text { General equation } \\
\text { for ellipse }
\end{array} x=\sqrt{\frac{r^{2}-y^{2}(\mathrm{I}-e)}{(\mathrm{I}-e)^{2}}} \\
& \text { For Cornea } e=0.5 \\
& r=7.8 \mathrm{~mm} \text {. } \\
& \therefore \text { Equation is } x=\sqrt{243^{\cdot} 3^{6-2 y^{2}}}
\end{aligned}
$$

F I G. I Wide-angle schematic anatomical eye showing technique of ray tracing through the aspheric surface

The tangential value was detirmined for the modified schematic eye by the following equation:

$$
\mathcal{T}=\frac{\mathrm{II} \cdot 06(\sin 5 \cdot 18 \mathrm{I} d)}{57 \cdot 3 \sin \theta}
$$

Where $T$ is the tangential dimension on the retina corresponding to a coaxial arc of $I^{\circ}$ length in visual space, at a peripheral angle of $\theta^{\circ}$ from the optic axis, and $d$ is the polar eccentricity of the image point in millimetres of retinal arc. The product of the retinal dimensions corresponding to a radial and tangential degree was considered to equal the area of retina corresponding to one solid degree at a given peripheral angle from the optical axis. The data were then transformed to visual axis data by correction for angle alpha.

\section{Results}

The non-linear function of radial eccentricity $d$, of a retinal point corresponding to an external peripheral angle $\theta$ from the optic axis is shown in Fig. 2. It was found to be in close agreement with the original data of Stine (r934) for peripheral angles of less than $60^{\circ}$, beyond which point the ellipsoid cornea produced an increasing departure from the spherical case.

This amounted to $3^{\circ}$ at the limits of the visual field. Similarly, the data on nodal point prediction appeared to coincide for angles of less than $55^{\circ}$. Data on the internal angle are shown in Fig. 3 for comparison with those of Lotmar (197I) using more complex optics, and are seen to be in close agreement. From the data plotted in Fig. 2, the values for radial magnification were obtained by interpolation (Fig. 4). The values of tangential magnification are seen to be non-linear to a fairly similar extent (Fig. 4), and those on area projection are consequently markedly so, as shown in Fig. 5 .

\section{Discussion}

The variation of the retinal projection of a solid degree is substantial and has several interesting consequences. Retinal cell counts cannot be accurately related to the visual field without the use of such data and the apparent size of the retinal lesion seen ophthalmoscopically is similarly distorted. The decreased area projection (see Fig. 5) reaches 37 per 

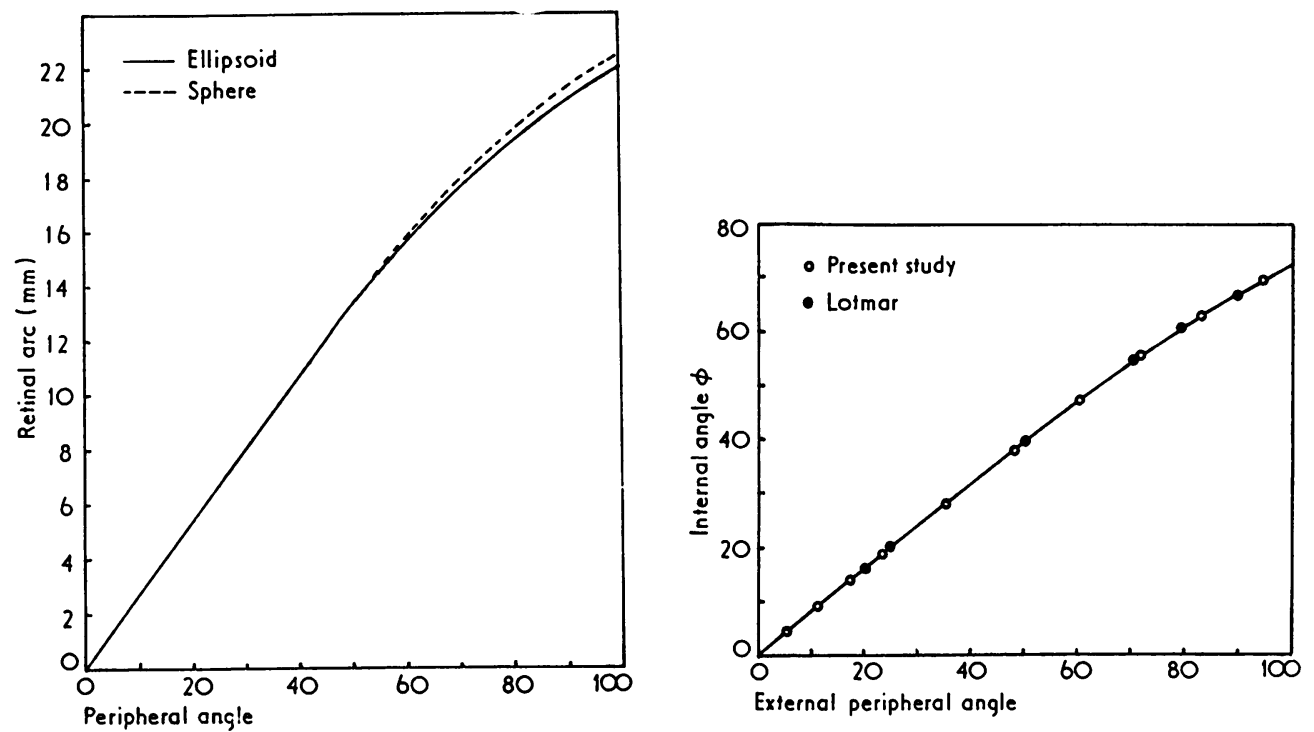

FIG. 2 Curve showing computed relationship between retinal arc lengths and visual angles from the optic axis FIG. 3 Data on external and internal peripheral angles of the simplified eye and the more complex model of Lotmar
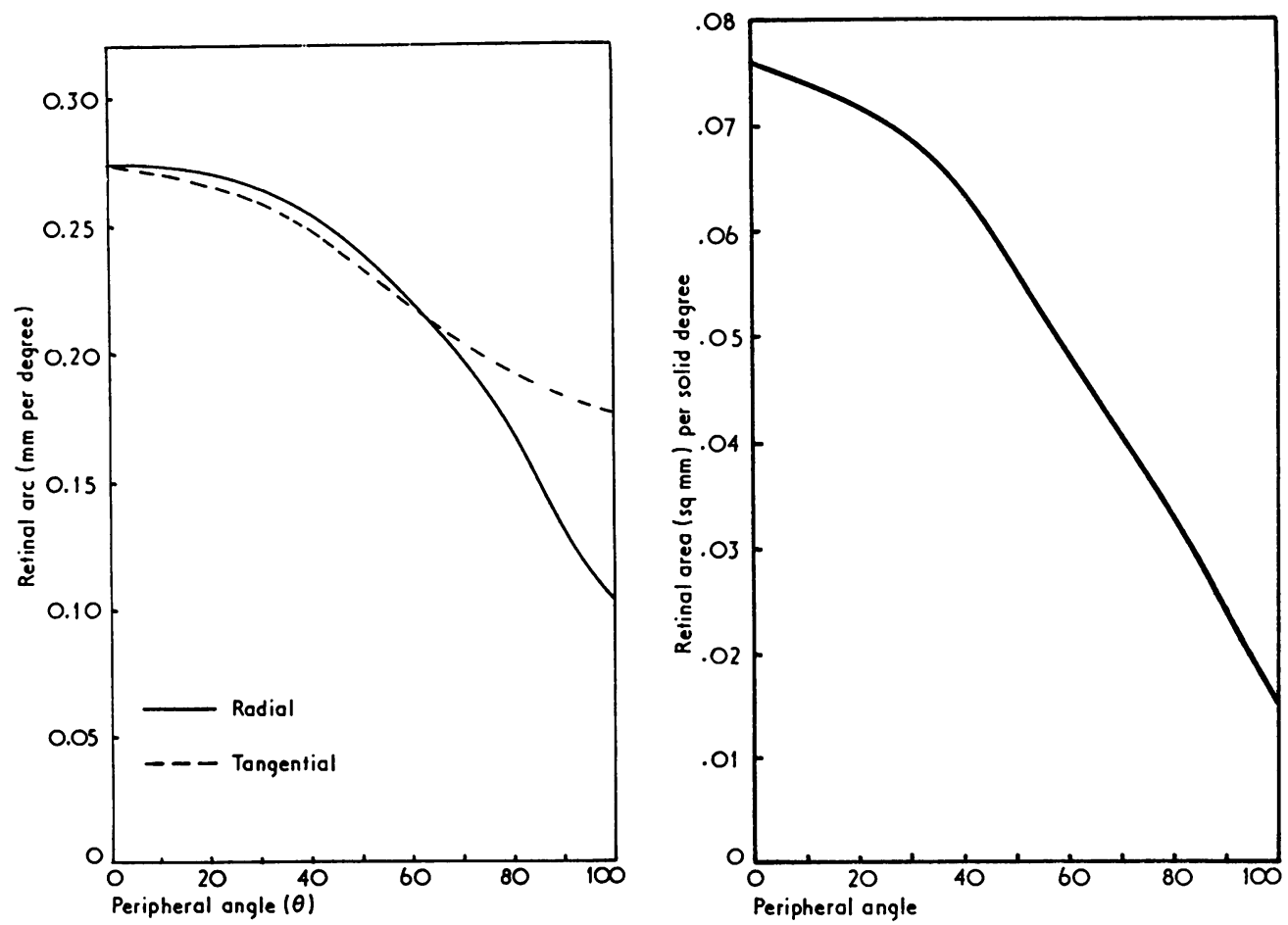

FIG. 4 Variation of radial and tangential magnification with peripheral angle from the optic axis

FIG. 5 Variation of retinal area per solid degree with peripheral angle from the optic axis 
cent. of the foveal value in the $80^{\circ}-90^{\circ}$ region. The size of the retinal area corresponding to a scotoma shown on a conventional isometric perimeter chart is subject to even greater distortion, as may be seen in Fig. 6. The projection of the visual field in terms of retinal area is represented in this chart and the area distortion of the conventional chart is shown. It exceeds 400 per cent. in the $80^{\circ}-90^{\circ}$ region.

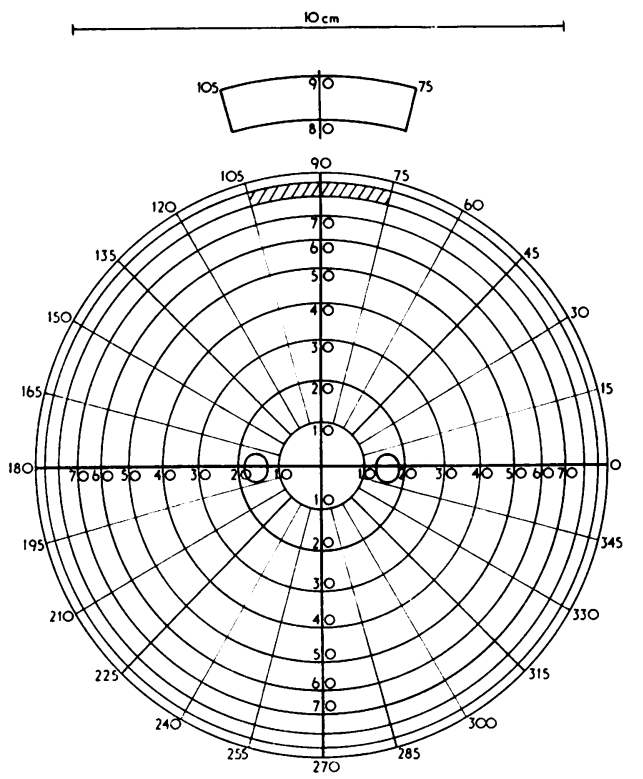

FIG. 6 Non-linear projection of the vusual field in which the retinal area is correctly represented. The section of the conventional isometric chart shows its area distortion when compared with the corresponding hatched region. The absolute value of a retinal area in sq. mm. is found by multiplying the measured area on the chart in sq. $\mathrm{cm}$. by 10

The retinal area corresponding to a scotoma may be assessed directly by plotting on the chart and measuring by planimetry or grid. This may prove useful in photocoagulation studies (Taylor, 1970), or other situations in which quantitative data are required on the size of peripheral retinal areas. Similarly, the total retinal area which has been the subject of recent deliberations (Taylor and Jennings, I97 I) may be assessed and this serves as an additional check on the principles involved. It is found to agree with our directly calculated values for the eye which are $\mathrm{I}, 065 \mathrm{sq} . \mathrm{mm}$. for the total retina excluding the disc and $\mathrm{I}, 020$ sq. $\mathrm{mm}$. for the functional retina excluding the disc and pars caeca.

These values are simply determined using the following formula for the area of the major segment of the sphere:

$$
A=(\mathrm{I}+\cos \rho) 2 \pi r^{2}
$$

where the supplementary angle $\left(180^{\circ}-\rho\right)$ is the average angular extent of the retina for all quadrants (see Fig. I and the Table).

The result is comparable to that of Taylor and Jennings (I97 I), who used a more complex method and obtained a value of $I_{1} 3^{2} \mathrm{sq}$. $\mathrm{mm}$. from rather similar anatomical data. An apparently neglected source of area measurement is the work of Van Buren (1963), who found a value of 943.5 sq. $\mathrm{mm}$. after correction for shrinkage on six human retinae, originating from eyes with diverse refractive errors. Since the measurement of area is a square function, it is highly sensitive to variation, and the values of Van Buren's small sample can be considered reasonably consistent with our findings. This discrepancy must also be balanced against opposing evidence from a recent major topographical study of the retina (Straatsma, 
Foos, and Spencer, I969), which showed slightly larger linear dimensions than those used in this calculation.

It seems probable that the variations in ocular dimensions found in different studies are due to sample construction rather than to measurement error. The influence of the changing regional differences in social conditions and genetic composition and the methods of sampling involved in the use of post mortem or other material may account for conflicting results. No single study is universally valid, but the dimensions selected for this paper are considered to be a reasonable compromise.

\section{Summary and conclusions}

The projection of the visual field on the retina of a schematic eye with an aspherical cornea has been investigated by trigonometrical ray tracing. Data on the radial image size as a function of peripheral angle differ slightly from those of an earlier study. New data on tangential and area projection also show marked variation with eccentricity.

In the peripheral retina, the area corresponding to a solid degree is shown to reach half of the foveal value. This is related to the size of the lesion viewed ophthalmoscopically, and is a source of error in existing data on the spatial projections of retinal cell densities. The effect is further exaggerated by the conventional perimeter chart, which gives rise to an area distortion of 400 per cent. in the $80^{\circ}$ to $90^{\circ}$ region when relating the plotted scotoma area to the receptive area of the retina.

We wish to acknowledge the support of the Science Research Council for the study on binocular vision which gave rise to some of the work upon which this paper is based.

\section{References}

cowan, A. (1928) "Ophthalmic Optics", pp. I53-1 74. Davies, Philadelphia

DUKE-ELDER, S., and ABRAMS, D. (1970) "System of Ophthalmology", vol. 5, "Ophthalmic Optics an d Refraction", p. 96. Kimpton, London

— and wYBar, K. (I96I) Idem, vol. 2, "The Anatomy of the Visual System", pp. 80-287

неLмногтZ, н. ( 1866 ) "Handbuch des physiologischen Optik", trans. from 3 rd German ed. by J.P.C.

Southall, vol. 2, p. 324 (1924). Optical Society of America. Dover Publications, New York

Listing, J. B. (1853) Cited by Duke-Elder and Abrams (1970, p. 12 I)

lotmar, w. (1971) F. opt. Soc. Amer., 61, I522

MANDEll, R. B., and ST HELEN, R. (1971) Brit. F. physiol. Optics, 26, 183

PARKer, J. A. (1972) Canad. 7. Ophthal., 7, I68

pomerantZeff, o., govignon, J., and schepens, c. L. (197i) Ann. Ophthal., 3, 8 I 5

PREChtel, L. A., and Wesley, N. K. (1970) Brit. F. physiol. Optics, 25, i I 7

STine, G. H. (1934) Amer. F. Ophthal., 17, 314

straAtsma, B. R., FOos, R. Y., and SPEncer, L. M. (r969) "Symposium on Retinal Surgery", pp.

I-26. Mosby, St. Louis

TAYLOR, E. (1970) Brit. F. Ophthal., 54, 535

- and JENNINGs, A. (1971) Ibid., 55, 262

van buRen, J. M. (1963) “The Retinal Ganglion Cell Layer”. Thomas, Springfield, Ill. 